\title{
Intravitreal Ranibizumab or Aflibercept After Bevacizumab in Diabetic Macular Edema: Exploratory Retrospective Analysis
}

This article was published in the following Dove Press journal:

Clinical Ophthalmology

\author{
Bernardete Pessoa (1D ${ }^{1,2}$ \\ Luísa Malheiro (D) \\ Inês Carneiro' \\ Sílvia Monteiro (D) \\ João Coelho id \\ Constança Coelho iD $^{3}$ \\ João Figueira (iD ${ }^{4-6}$ \\ Angelina Meireles (D) ${ }^{1,2}$ \\ João Nuno Melo Beirão ${ }^{1,2}$ \\ 'Ophthalmology Department, Centro \\ Hospitalar e Universitário do Porto, \\ Porto, Portugal; ${ }^{2}$ Instituto de Ciências \\ Biomédicas Abel Salazar, Universidade do \\ Porto, Porto, Portugal; ${ }^{3}$ Genetics \\ Laboratory, Institute of Environmental \\ Health, Lisbon Medical School, University \\ of Lisbon, Porto, Portugal; ${ }^{4}$ Centro \\ Hospitalar e Universitário de Coimbra, \\ Porto, Portugal; ${ }^{5}$ Faculty of Medicine of \\ the University of Coimbra, Porto \\ Portugal; ${ }^{6}$ Association for Innovation and \\ Biomedical Research on Light and Image, \\ Coimbra, Portugal
}

Correspondence: Bernardete Pessoa Centro Hospitalar e Universitário do Porto - Hospital de Santo António, Departamento de Oftalmologia, Largo Prof. Abel Salazar, Edifício Neoclássico, Porto 4099-00I, Portugal

Tel +35I 938469884

Email bbtpessoa@gmail.com
Aim: To evaluate the efficacy of switching from bevacizumab to ranibizumab or aflibercept in eyes with diabetic macular edema (DME) unresponsive to bevacizumab.

Methods: Single-center retrospective comparative study of patients with DME unresponsive to intravitreal bevacizumab that was switched to ranibizumab or aflibercept. Best-corrected visual acuity (BCVA) and central foveal thickness (CFT) were analysed prior to and 4 months after the switch. Ocular coherence tomography (OCT) biomarkers were also analysed.

Results: Fifty-six eyes from 40 patients were included in the study, 33 eyes switched to ranibizumab and 23 to aflibercept. A significant median CFT decrease was observed in both groups ( $p<0.001$ ), with no between-group differences. BCVA gain was only significant in the ranibizumab group $(p<0.001)$. None of the pre-baseline or baseline parameters were associated with the response to ranibizumab or aflibercept.

Conclusion: In persistent DME unresponsive to bevacizumab, both anatomical and functional improvements were observed with ranibizumab whereas aflibercept only showed an anatomical improvement. Clinicaltrials.gov NCT04018833.

Keywords: aflibercept, bevacizumab, diabetic macular edema, ranibizumab, refractory

\section{Introduction}

Diabetic retinopathy (DR) is the leading cause of vision loss among working-age adults in the developed world, ${ }^{1}$ and diabetic macular edema (DME) is the main responsible for the vision loss related to DR.

The International Council of Ophthalmology and EURETINA Guidelines recommend anti-vascular endothelial growth factors (anti-VEGF) agents, ranibizumab $^{2,3}$ and aflibercept, ${ }^{4}$ as well as off-label bevacizumab, ${ }^{5}$ as first-line therapy for treating central DME., ${ }^{6,7}$ The lower cost of bevacizumab, perceived effectiveness and relative safety, makes it a widely accepted therapy option particularly in the developing world. ${ }^{8}$ Protocol $\mathrm{T}$ is the only head-to-head study in DME with the three anti-VEGF agents. ${ }^{9}$ However, ranibizumab was used at $60 \%(0.3 \mathrm{mg})$ of the dose approved by the European Medicines Agency (EMA), which is $0.5 \mathrm{mg}{ }^{2}$ Although in the first 3 years of the RIDE and RISE (A Study of Ranibizumab Injection in Subjects With Clinically Significant Macular Edema (ME) With Center Involvement Secondary to Diabetes Mellitus, NCT00473382 and NCT0047330, respectively) trials no statistically significant difference in functional or anatomical outcomes has been detected between the $0.3 \mathrm{mg}$ and the $0.5 \mathrm{mg}$ doses, their 
comparison was not performed under a pro re nata (PRN) regimen. In fact, in an extended dose regimen strategy, those differences could have emerged. ${ }^{9}$ In more severe or refractory cases, this is the rational for switching from a less effective off-label anti-VEGF to ranibizumab or aflibercept.

There are numerous data showing the efficacy of ranibizumab or aflibercept for the treatment of patients with DME refractory to bevacizumab, as well as with an antiVEGF switch approach in cases where tachyphylaxis or tolerance to a previously effective anti-VEGF may occur. ${ }^{10-12}$ Much less evidence exists on the real efficacy differences between aflibercept versus ranibizumab treatment after an initial bevacizumab regimen. ${ }^{13}$

Retinal edema is responsible for retinal microstructural changes, retinal atrophy of photoreceptors and ganglion cell lesions. ${ }^{14}$ It may also be considered consensual that the best improvements in visual acuity are achieved when retinal edema is resolved. In a background of a chronic and progressive disease, DME should be faced as a condition to control as efficiently and promptly as possible. Therefore, it is crucial to identify early nonresponders to an anti-VEGF considering also an early therapeutical switch with the purpose of achieving the best anatomical and functional outcomes. The key to an early identification of a nonresponder lies on the identification of prognostic biomarkers. According to the literature, the presence of OCT biomarkers such as subretinal fluid (SRF), ellipsoid layer disruption (ELd), external limiting membrane disruption (ELMd), hyperreflective foci (HRF), cysts in the outer nuclear layer (ONLc) and their size, hard exudates (HE) and disorganization of the retinal inner layers (DRIL) are correlated with a more severe, chronic DME and poor retina function but not with an expected negative anti-VEGF response. ${ }^{15-19}$ Only the presence of outer plexiform layer disruption (OPLd), epiretinal membrane (ERM) with retina wrinkling and the loss of deep capillary plexus in OCT angiography have shown to be predictive of a poor response to anti-VEGF. ${ }^{15-19}$

A vitreomacular detachment from the macular area (VMA) with some possible anterior-posterior traction (considered in this study when at least one-third of VMA was present) may also play a role in DME evolution, particularly in treatment response. The tangential as well as anterior-posterior traction, through a synergic effect, may contribute to a higher hydrostatic pressure towards the retina tissue..$^{20-22}$
The purpose of this study was to evaluate the safety and efficacy of switching to ranibizumab or aflibercept in cases of DME refractory to off-label bevacizumab treatment, and to identify any parameters associated with positive or negative prognosis following this switch.

\section{Subjects, Materials and Methods Study Design}

This was a single-center retrospective comparative study of consecutive DME cases unresponsive or incompletly responsive to intravitreal (IV) bevacizumab $(1.25 \mathrm{mg} /$ $0.05 \mathrm{~mL})$ that were switched to IV ranibizumab $(0.5 \mathrm{mg} /$ $0.05 \mathrm{~mL})$ or aflibercept $(2.0 \mathrm{mg} / 0.05 \mathrm{~mL})$.

The study was conducted according to the tenets of the Declaration of Helsinki in its latest amendment (Brazil, 2013) as was approved by the Ethics Committee of HSACHUP. All patients signed an informed consent prior to entering the study. This study is registered on clinicaltrials. gov under the number NCT04018833.

\section{Setting and Participants}

The study was performed at Hospital de Santo António-Centro Hospitalar e Universitário do Porto (HSA-CHUP), Portugal.

The clinical records of 188 eyes from 128 patients with DME under IV treatment between January 2012 and October 2015 were reviewed. A total of 147 eyes were identified as having started IV treatment for DME with bevacizumab.

Inclusion criteria were patients with type 1 or type 2 diabetes mellitus, older than 18 years, with center-involved DME, defined as central foveal thickness (CFT) of more than $300 \mu \mathrm{m}$ on spectral-domain OCT (SD-OCT). All patients included were considered nonresponsive to bevacizumab, defined as having persistent intraretinal and/or subretinal fluid on OCT, ie, CFT $>300 \mu \mathrm{m}$ and $\leq 10 \%$ CFT decrease from the last two consecutive bevacizumab IV, after a minimum of 3 monthly injections, with CFT assessed during the fourth week after the last bevacizumab injection before switching, regardless of visual acuity (VA).

Exclusion criteria included additional ocular diseases that could significantly affect the VA: a significant vitreoretinal interface anomaly on SD-OCT that might contribute to macular edema, such as an ERM with inner retinal distortion including proliferative diabetic retinopathy (PDR) with tractional retinal detachment or vitreous haemorrhage; age-related macular degeneration; retinal vascular occlusion; central corneal opacity; amblyopia; advanced glaucoma; 
optic neuropathy; vitreous opacity; history of ocular trauma or surgery other than uncomplicated cataract extraction; cataract surgery within 6 months before bevacizumab switch; and inability or unwillingness to provide informed consent. Previous vitrectomy was not considered an exclusion criteria if DME occurred after a minimum period of 6 months post-vitrectomy. Both eyes of a single patient could be included only if both met all of the inclusion criteria, none of the exclusion criteria, and if the patient was switched to the same anti-VEGF at the time.

\section{Methods of Assessment}

Demographic characteristics, type of diabetes, presence or absence of diabetic retinopathy, HbA1c, CFT, BCVA in Early Treatment Diabetic Retinopathy Study (ETDRS) letters, and comorbidities were collected from the clinical records before starting bevacizumab injections (prebaseline).

Baseline was considered as the time point immediately before the switch. At this time, collected variables were previous laser therapy, number of previous bevacizumab IV injections, CFT and BCVA. Moreover, we have considered as OCT biomarkers the presence of 10 different morphological parameters (Figure 1A and B), six in $1 \mathrm{~mm}$ central foveal area and four within the $20 \times 20^{\circ}$ scan area. In the $1 \mathrm{~mm}$ central foveal area: 1) SRF presence; 2) OPLd; 3) DRIL; 4) ELd; 5) ELMd; 6) existence of more than $10 \mathrm{HRF}$. Within the $20 \times 20^{\circ}$ scan area: 7) cysts in the outer nuclear layer more expressive than in the inner nuclear layer (ONLc>INLc); 8) HE evidence; 9) ERM without inner retinal distortion (ERMn); and 10) vitreomacular adhesion with at least one-third of VMA.
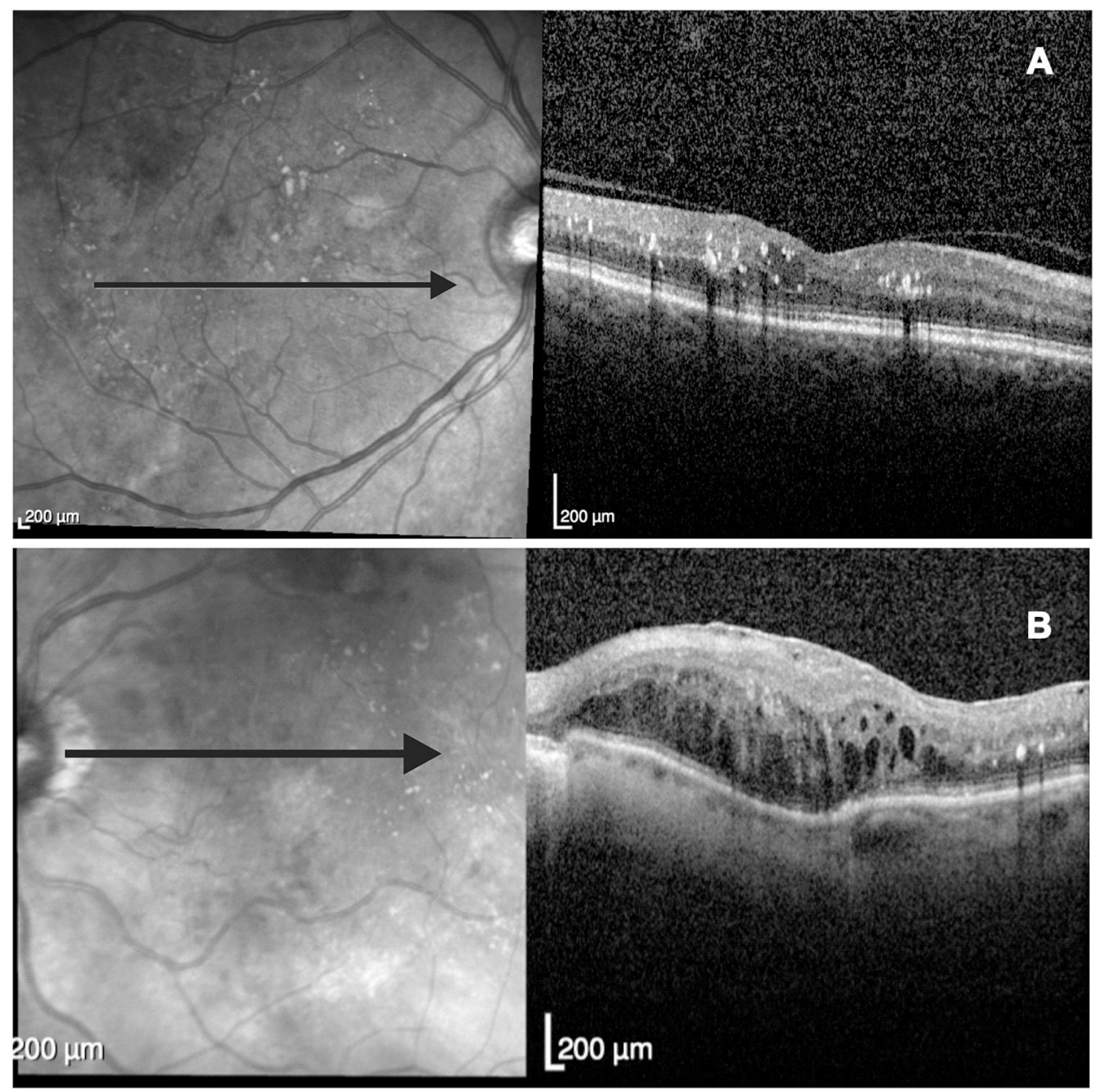

Figure I (A) Example of a central foveal image of an OCT 20x20 scan area (5.8 mm) acquisition where the existence of hard exudates (HE); hyperreflective foci (HRF) with small size $(<30 \mu \mathrm{m})$, with reflectivity similar to the nerve fiber layer and no back-shadowing; hard exudates (HE) with back-shadowing; and a vitreomacular adhesion with at least I/3 of vitreomacular detachment from the macular area (VMA) can be observed. (B) Example of a central foveal image of an OCT $20 \times 20^{\circ}$ scan area acquisition with the presence of an epiretinal membrane without inner retinal distortion (ERMn); outer plexiform layer disruption (OPLd); disorganization of the retinal inner layers (DRIL); ellipsoid layer disruption (ELd); external limiting membrane disruption (ELMd); HE; and HRF. 
A single $180^{\circ}$ SD-OCT line scan (5.8mm length) centered onto the fovea was analyzed for HRF evaluation. A manual count of HRF, defined as small $(<30 \mu \mathrm{m})$, punctiform, with reflectivity similar to the nerve fiber layer and no backshadowing, was performed in the central $1 \mathrm{~mm}$ in length.

After the switch, all patients received three monthly consecutive doses of ranibizumab or aflibercept and were observed during the fourth week after the last injection, when CFT and BCVA were assessed.

OCT scans were obtained by an SD-OCT (macular dense line scan mode HR 20x20 Spectralis HRA+OCT; Heidelberg Engineering, Heidelberg, Germany). The OCT data evaluation was performed by two experienced medical retina specialists, $\mathrm{BP}$ and $\mathrm{MB}$, and the mean value of these measurements was considered.

The CFT was automatically measured by the software in the central $1 \mathrm{~mm}$. A CFT $<300 \mu \mathrm{m}$ was defined as DME resolution. After switching, during the fourth week after the last injection, a clinically significant functional improvement was considered when a gain of $\geq 5$ EDTRS letters was achieved and a clinically significant anatomical improvement was considered if a reduction in CFT $\geq 10 \%$ was achieved.

\section{Quantitative Variables}

Quantitative variables reported on this paper are $\mathrm{HbA1c}$, CFT, and BCVA in ETDRS letters.

\section{Statistical Methods}

Non-parametric statistical methods were used. Values are presented as median (range, interquartile range) or $\mathrm{n}(\%)$ unless otherwise specified. Within-group analyses were performed with the Friedman or Wilcoxon tests for paired samples, depending on the number of time points analysed. The McNemar test was used for discrete variables. Between-group analyses (ranibizumab versus aflibercept) at each time point were performed with the MannWhitney test. The $x^{2}$ test was used for comparison of proportions. Whenever necessary, p-values were adjusted for multiple comparisons. Data analyses were performed using SPSSv23 (IBM., USA). Tests were considered significant at $\alpha<0.05$ significance level (two-sided).

\section{Results}

\section{Participants}

A total of 56 eyes from 40 patients were included in the study.

\section{Demographics and Clinical Baseline Characteristics}

Demographic, pre-baseline and baseline clinical data from the total population sample and patients that switched to ranibizumab or aflibercept are shown in Table 1. There were no between-group statistically significant differences in demographic characteristics, pre-baseline or baseline parameters, except for DRIL presence in baseline OCT parameters - Table 2. There were more eyes without DRIL in the ranibizumab group than in the aflibercept group $(63.6 \%$ vs $26.1 \%$, $\mathrm{p}=0.007)$.

\section{Between- and Within-Group Anatomical Differences Post-Switch}

Median CFT decrease was significantly different in both switch groups after 4 months both compared to prebevacizumab and to the moment of the switch $(p<0.001)$, with no between-group differences - Figure 2 .

\section{Between- and Within-Group Functional Differences Post-Switch}

BCVA gain was only statistically significant in the postswitch ranibizumab group after 4 months, both compared to pre-bevacizumab and the moment of the switch $(p<0.001)$ - Figure 3. Moreover, more patients increased more than 15 letters in the ranibizumab group compared with the aflibercept group $(18.2 \%$ vs $0 \%$, respectively, $\mathrm{p}=0.037$ ) after 4 months of the switch.

\section{Discussion}

This single-center retrospective comparative study describes the efficacy of switching from bevacizumab to ranibizumab or aflibercept in eyes with DME unresponsive to bevacizumab. Of the 40 patients included in the study 56 eyes were analyzed.

The only difference between groups, among baseline characteristics, was the presence of more eyes without DRIL in the ranibizumab group which may explain the BCVA gain differences between groups, with only a statistically significant improvement in the ranibizumab group. Following the same pattern, more eyes had an increase of more than 15 letters in the ranibizumab group compared with the aflibercept group. Similar outcomes have been already described by Ashraf et $\mathrm{al}^{13}$ who reported that despite a significant CFT decrease after the switch to ranibizumab or aflibercept in eyes with DME refractory to bevacizumab, a significant improvement in 
Table I Demographics, Pre-Baseline and Baseline Clinical Characteristics for the Whole Population and by Switch Group (Ranibizumab or Aflibercept)

\begin{tabular}{|c|c|c|c|c|}
\hline Parameters & $\begin{array}{l}\text { Whole Population Sample } \\
(n=56)\end{array}$ & $\begin{array}{l}\text { Switch to Ranibizumab } \\
(n=33)\end{array}$ & $\begin{array}{l}\text { Switch to Aflibercept } \\
(n=23)\end{array}$ & p-value* \\
\hline Age in years, median (range, IQR) & $67.0(47.0-85.0,11.0)$ & $65.5(47.0-85,11.0)$ & $69.0(52.0-81.0,14.0)$ & 0.648 \\
\hline HT, n (\%) & $28(77.8)$ & $13(65.0)$ & $15(93.8)$ & 0.053 \\
\hline Dyslipidemia, n (\%) & $24(66.7)$ & II $(55.0)$ & $13(81.2)$ & 0.157 \\
\hline BMI, median (range, IQR) & $27.8(20.6-40.2,5.1)$ & $27.3(20.6-35.0,7.6)$ & $27.9(24.0-40.2,10.0)$ & 0.300 \\
\hline Type 2 DM, n (\%) & $39(69.6)$ & $24(72.7)$ & $15(65.2)$ & 0.400 \\
\hline DM duration in months, median (range, IQR) & $16.0(0.0-32.0,10.0)$ & $15.5(0.0-31.0,16.0)$ & $18.0(5.0-32.0,8.0)$ & 0.705 \\
\hline DME duration in months, median (range, IQR) & $10.3(0.0-48.0,12.0)$ & $8.5(0.0-36.0,9.0)$ & $12.9(1.0-48.0,22.0)$ & 0.842 \\
\hline PDR, n (\%) & $2(3.6)$ & I (3.0) & $\mathrm{I}(4.3)$ & 1.000 \\
\hline HbAlc in \%, median (range, IQR) & $7.9(6.0-9.0,3.0)$ & $7.8(6.0-8.8,0.8)$ & $7.9(6.7-9.0,2.3)$ & 0.606 \\
\hline Phakic, n (\%) & $48(85.7)$ & $30(90.9)$ & $18(78.3)$ & 0.252 \\
\hline Pre-switch macular laser, $\mathrm{n}(\%)$ & $12(21.8)$ & $7(21.9)$ & $5(21.7)$ & 1.000 \\
\hline Pre-switch PRP laser, n (\%) & $14(25.9)$ & II (35.5) & $3(13.0)$ & 0.115 \\
\hline $\begin{array}{l}\text { Number of pre-switch beva injections, } \\
\text { median (range, IQR) }\end{array}$ & $3.0(3.0-14.0,3.0)$ & $3.0(3.0-14.0,2.0)$ & $4.0(3.0-11.0,3.0)$ & 0.231 \\
\hline Pre-beva CFT in $\mu \mathrm{m}$, median (range, IQR) & $473.0(320.0-808.0,119.0)$ & $483.0(320.0-808.0,78.0)$ & $459.0(332.0-644.0,136.0)$ & 0.191 \\
\hline Pre-switch CFT in $\mu \mathrm{m}$, median (range, IQR) & $468.5(312.0-707.0,131.0)$ & $449.0(312.0-707.0,117.0)$ & $473.0(331.0-603.0,153.0)$ & 0.868 \\
\hline Pre-beva BCVA, median (range, IQR) & $60.0(3.0-80.0,25.0)$ & $60.0(3.0-80.0,20.0)$ & $60.0(10.0-80.0,25.0)$ & 0.712 \\
\hline Pre-switch BCVA, median (range, IQR) & $65.0(3.0-85.0,25.0)$ & $65.0(3.0-85.0,23.0)$ & $61.0(10.0-80.0,27.0)$ & 0.893 \\
\hline $\begin{array}{l}\text { Time in months from last beva to first } \\
\text { post-switch injection, median (range, IQR) }\end{array}$ & $3.1(1.0-9.0,3.0)$ & $3.0(1.0-9.0,3.0)$ & $3.3(1.0-8.0,2.0)$ & 0.434 \\
\hline
\end{tabular}

Note: *p-value between ranibizumab and aflibercept groups.

Abbreviations: IQR, interquartile range; DM, diabetes mellitus; HT, hypertension; BMI, body mass index; DME, diabetic macular edema; PDR, proliferative diabetic retinopathy; HbAIc, glycated hemoglobin; PRP, photocoagulation; beva, bevacizumab; CFT, central foveal thickness; BCVA, best-corrected visual acuity in ETDRS letters.

Table 2 OCT Parameters for the Whole Population and by Switch Group (Ranibizumab or Aflibercept)

\begin{tabular}{|c|c|c|c|c|}
\hline $\begin{array}{l}\text { OCT } \\
\text { Parameters }\end{array}$ & $\begin{array}{l}\text { Whole Population Sample } \\
(n=56)\end{array}$ & $\begin{array}{l}\text { Switch to Ranibizumab } \\
(n=33)\end{array}$ & $\begin{array}{l}\text { Switch to Aflibercept } \\
(n=23)\end{array}$ & p-value* \\
\hline SRF, n (\%) & $6(10.7)$ & $4(12.1)$ & $2(8.7)$ & 1.000 \\
\hline OPLd, n (\%) & 49 (87.5) & 27 (8I.8) & $22(95.7)$ & 0.220 \\
\hline DRIL, n (\%) & $29(51.8)$ & $12(36.4)$ & 17 (73.9) & 0.007 \\
\hline ELd, n (\%) & $27(48.2)$ & $13(39.4)$ & $14(60.9)$ & 0.174 \\
\hline ELMd, n (\%) & $24(42.9)$ & II (33.3) & I3 (56.5) & 0.105 \\
\hline ONL>INL, n (\%) & $29(51.8)$ & $17(5 \mid .5)$ & $12(52.2)$ & 1.000 \\
\hline HE, n (\%) & $36(64.3)$ & $19(57.6)$ & 17 (73.9) & 0.264 \\
\hline > 10 HRD, n (\%) & $53(94.6)$ & $30(90.9)$ & $23(100.0)$ & 0.261 \\
\hline ERMn, n (\%) & $17(30.4)$ & II (33.3) & $6(26.0)$ & 0.734 \\
\hline VMA, n (\%) & $9(16.1)$ & $3(9.1)$ & $6(26.1)$ & 0.139 \\
\hline
\end{tabular}

Note: *p-value between ranibizumab and aflibercept groups.

Abbreviations: OCT, optical coherence tomography; SRF, subretinal fluid; OPLd, outer plexiform layer disruption; DRIL, disorganization of the retinal inner layers; ELd, ellipsoid layer disruption; ELMd, external limiting membrane disruption; ONL>INL, cysts in the outer nuclear layer more expressive than in the inner nuclear layer; HE, hard exudates; > 10 HRD, more than 10 hyperreflective dots; ERMn, epiretinal membrane without inner retinal distortion; VMA, vitreomacular adhesion with at least I/3 of vitreomacular detachment from the macular area.

BCVA was observed only with ranibizumab but not with aflibercept. As it is already well established, there is an association between DRIL, increasing severity of diabetic retinopathy, and a poor VA outcome with treatment. ${ }^{23,24}$ In our cohort, the higher prevalence of baseline DRIL in the aflibercept group did not parallel a lower baseline visual acuity in comparison with the ranibizumab group. Until now, the influence of OCT biomarkers, such as DRIL, on functional outcomes with anti-VEGF therapy, has not been addressed in hallmark clinical trials such as protocol T. ${ }^{9}$

Protocol T, the only head-to-head study comparing the effect of the three anti-VEGFs in DME, corroborates these 


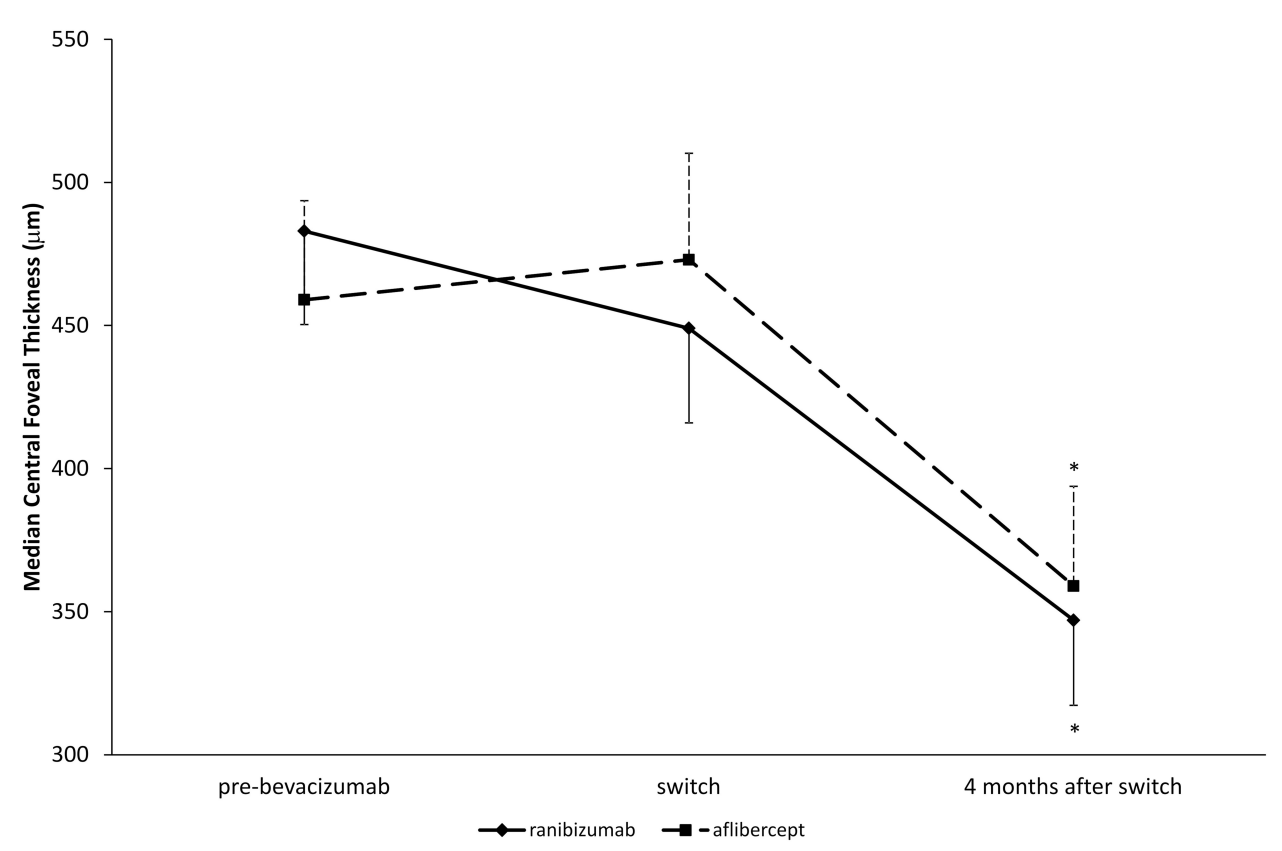

Figure 2 Median central foveal thickness change between- and within- switch groups (ranibizumab or aflibercept) pre-bevacizumab, at the time of switch and 4 months after switch. ${ }^{*}<<0.001$ compared both to pre-bevacizumab and to switch (within-group).

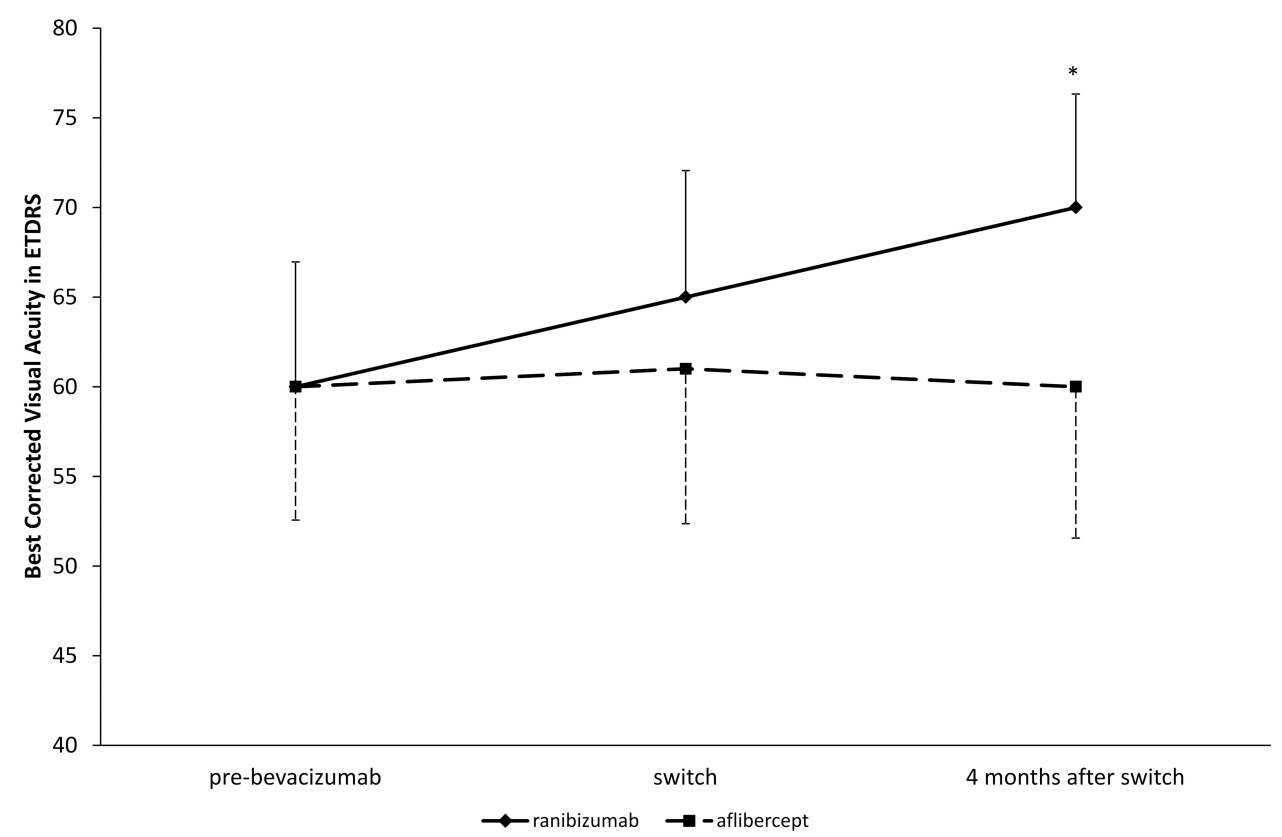

Figure 3 Best-corrected visual acuity change between- and within- switch groups (ranibizumab or aflibercept) pre-bevacizumab, at the time of switch and 4 months after switch. ${ }^{*} p<0.001$ compared compared both to pre-bevacizumab and to switch (within-group).

findings, although with a $0.3 \mathrm{mg}$ ranibizumab dose (which has not been tested against the $0.5 \mathrm{mg}$ dose under a PRN regimen). In the United States ranibizumab, $0.3 \mathrm{mg}$ is only approved for DME treatment in a monthly regimen and not in a PRN regimen. ${ }^{3}$ In fact, the Resolve Phase II study ended with a target average treatment dose of $0.47 \mathrm{mg}^{25}$
Interestingly, and even using a possible suboptimal ranibizumab dose whilst using the recommended aflibercept dose, the 2 years results of protocol $\mathrm{T}$ have shown that there were no statistically significant differences between ranibizumab and aflibercept, regarding CFT decrease and BCVA improvement. On the contrary, 
bevacizumab was less efficient in comparison with ranibizumab and aflibercept in achieving a BCVA gain in patients with less than 69 letters at baseline and in reducing macular edema (even in patients with a good baseline BCVA, $>69$ letters). ${ }^{9}$ Our results reinforce the conclusion of protocol $\mathrm{T}$ in as much as a sub-optimal response to bevacizumab was verified in patients with prebevacizumab low vision, $<69$ letters, and our study population had a mean BCVA lower than 69 letters in both pre-bevacizumab and pre-switch time points.

Favoring our study design with early switch criteria is the post-hoc analysis of the DRCR.net Protocol I. ${ }^{26}$ According to this study, and for patients with a suboptimal visual response after the first 3 IV anti-VEGF injections, it may be appropriate to consider adjustments to the treatment regimen and an early switch to achieve the best functional outcomes. Eyes with sub-optimal early BCVA response (after 12 weeks with monthly IV ranibizumab treatment) had poorer long-term visual outcomes than eyes with a pronounced early response. ${ }^{26}$

Following this line of reasoning, and although the average number of bevacizumab injections prior to switching (3-4) may be considered insufficient, it is possible that if patients had continued with bevacizumab treatment they might eventually improve. However, and in order to minimize this possibility, only patients with $\leq 10 \%$ CFT decrease from the last two consecutive bevacizumab IV were considered for switching in our cohort. The same rationale was used for the post-switch follow-up period.

In addition, the majority of studies accept structural criteria on OCT rather than functional outcomes as relevant for refractoriness. Although OCT may not be considered the best method to be used, it is still the most widely, reproducible and accessible method in real-world clinical practice. Moreover, a functional negative outcome is induced by previous structural retinal lesions such as DME.

The present study had limitations, such as its retrospective non-randomized nature, the small number of eyes, the absence of a control arm, the relatively short duration of follow-up, and the additional difficulty inherent to the multifactorial nature of DME.

This study also has strengths: our results were achieved using the same baseline anatomical and visual selection inclusion criteria for both groups, and therefore there is no selection bias; it reinforces that other possible, yet unidentified, anatomical macular biomarkers may influence the functional outcomes in response to different treatments. In our cohort, DRIL has emerged as an important biomarker to explore, but also other possible prognostic factors should be pursued, eg, macula vascularization profile through future, more accurate, and fully developed non-invasive imaging OCT technology such as OCT angiography (OCTA).

\section{Conclusion}

In persistent DME unresponsive or with incomplete response to bevacizumab, a significant anatomical and functional improvement was observed with ranibizumab therapy. In the aflibercept group, the anatomical response was not followed by an improvement on BCVA after 4 months of switch. Novel biomarkers, other than visual acuity and CFT outcomes, should be pursued, to clarify these achievements and the real efficacy of each individual therapy.

\section{Data Sharing Statement}

All data used to support the findings of this study are at the Ophthalmology Department, Hospital de Santo António, Centro Hospitalar Universitário do Porto, Portugal, and available from the corresponding author upon request. Any data intended for sharing is deidentified.

\section{Author Contributions}

All authors made a significant contribution to the work reported, whether that is in the conception, study design, execution, acquisition of data, analysis and interpretation, or in all these areas; took part in drafting, revising or critically reviewing the article; gave final approval of the version to be published; have agreed on the journal to which the article has been submitted; and agree to be accountable for all aspects of the work.

\section{Funding}

This research did not receive any specific grant from funding agencies in the public, commercial, or not-forprofit sectors.

\section{Disclosure}

The authors have no conflicts of interest to declare for this work.

\section{References}

1. Yau JW, Rogers SL, Kawasaki R, et al. Global prevalence and major risk factors of diabetic retinopathy. Diabetes Care. 2012;35 (3):556-564. doi:10.2337/dc11-1909

2. Summary of product characteristics ranibizumab. Approved by EMA. Last updated 2019. 2007. 
3. Summary of product characteristics ranibizumab. Approved by FDA revised 2014. 2006.

4. Summary of product characteristics aflibercept. Approved by EMA. Last updated 2019. 2012.

5. Summary of product characteristics bevacizumab. Approved by EMA. Last updated 2018. 2005.

6. International Council of Ophthalmology. ICO guidelines for diabetic eye care; 2017. Available from: http://www.icoph.org/downloads/ ICOGuidelinesforDiabeticEyeCare.pdf. Accessed January 12, 2021.

7. Schmidt-Erfurth U, Garcia-Arumi J, Bandello F, et al. Guidelines for the management of diabetic macular edema by the European Society of Retina Specialists (EURETINA). Ophthalmologica. 2017;237 (4):185-222. doi:10.1159/000458539

8. Hutton D, Newman-Casey PA, Tavag M, Zacks D, Stein J. Switching to less expensive blindness drug could save medicare part B $\$ 18$ billion over a ten-year period. Health Aff (Millwood). 2014;33(6):931-939. doi:10.1377/hlthaff.2013.0832

9. Wells JA, Glassman AR, Ayala AR, et al. Aflibercept, Bevacizumab, or Ranibizumab for diabetic macular edema: two-year results from a comparative effectiveness randomized clinical trial. Ophthalmology. 2016;123(6):1351-1359. doi:10.1016/j.ophtha.2016.02.022

10. Binder S. Loss of reactivity in intravitreal anti-VEGF therapy: tachyphylaxis or tolerance? $B r \quad J$ Ophthalmol. 2012;96(1):1-2. doi:10.1136/bjophthalmol-2011-301236

11. Katz G, Moisseiev E, Goldenberg D, et al. Ranibizumab for persistent diabetic macular edema after bevacizumab treatment. Eur J Ophthalmol. 2017;27(2):210-214. doi:10.5301/ejo.5000838

12. Rahimy E, Shahlaee A, Khan MA, et al. Conversion to aflibercept after prior anti-VEGF therapy for persistent diabetic macular edema. $\mathrm{Am}$ J Ophthalmol. 2016;164(118):e112. doi:10.1016/j.ajo.2015.12.030

13. Ashraf M, Souka AA, ElKayal H. Short-term effects of early switching to ranibizumab or aflibercept in diabetic macular edema cases with non-response to bevacizumab. Ophthalmic Surg Lasers Imaging Retina. 2017;48(3):230-236. doi:10.3928/23258160-20170301-06

14. Bressler SB, Ayala AR, Bressler NM, et al. Persistent macular thickening after ranibizumab treatment for diabetic macular edema with vision impairment. JAMA Ophthalmol. 2016;134(3):278-285. doi:10.1001/jamaophthalmol.2015.5346

15. Ashraf M, Souka A, Adelman R. Predicting outcomes to anti-vascular endothelial growth factor (VEGF) therapy in diabetic macular oedema: a review of the literature. $\mathrm{Br} J$ Ophthalmol. 2016;100(12):1596-1604. doi:10.1136/bjophthalmol-2016-308388

16. Maheshwary AS, Oster SF, Yuson RM, Cheng L, Mojana F, Freeman WR. The association between percent disruption of the photoreceptor inner segment-outer segment junction and visual acuity in diabetic macular edema. Am J Ophthalmol. 2010;150(1):63-67 e61. doi:10.1016/j.ajo.2010.01.039
17. Reznicek L, Cserhati S, Seidensticker F, et al. Functional and morphological changes in diabetic macular edema over the course of anti-vascular endothelial growth factor treatment. Acta Ophthalmol. 2013;91(7):e529-536. doi:10.1111/aos.12153

18. Sun JK, Radwan SH, Soliman AZ, et al. Neural retinal disorganization as a robust marker of visual acuity in current and resolved diabetic macular edema. Diabetes. 2015;64(7):2560-2570. doi:10.2337/db14-0782

19. Vujosevic S, Torresin T, Berton M, Bini S, Convento E, Midena E. Diabetic macular edema with and without subfoveal neuroretinal detachment: two different morphologic and functional entities. $\mathrm{Am}$ J Ophthalmol. 2017;181:149-155. doi:10.1016/j.ajo.2017.06.026

20. Karaca EE, Kepez Yldz B, Cubuk MO, Ozdek S. Epiretinal membranes in neovascular age-related macular degeneration: effect on outcomes of anti-vascular endothelial growth factor therapy. Retina. 2015;35(8):1540-1546. doi:10.1097/IAE.0000000000000531

21. Pessoa B, Coelho J, Coelho C, et al. Enzymatic vitreolysis for the treatment of tractional diabetic macular edema. Ther $A d v$ Ophthalmol. 2019;11:1-10.

22. Trevino A, Martinez MR, Ophir A. Chronic macular edema associated with extrafoveal vitreoretinal traction. Int $J$ Ophthalmol. 2010;3(4):321-325. doi:10.3980/j.issn.2222-3959.2010.04.10

23. Das R, Spence G, Hogg RE, Stevenson M, Chakravarthy U. Disorganization of inner retina and outer retinal morphology in diabetic macular edema. JAMA Ophthalmol. 2018;136(2):202-208. doi:10.1001/jamaophthalmol.2017.6256

24. Sun JK, Lin MM, Lammer J, et al. Disorganization of the retinal inner layers as a predictor of visual acuity in eyes with center-involved diabetic macular edema. JAMA Ophthalmol. 2014;132(11):1309-1316. doi:10.1001/jamaophthalmol.2014.2350

25. Massin P, Bandello F, Garweg JG, et al. Safety and efficacy of ranibizumab in diabetic macular edema (RESOLVE Study): a 12-month, randomized, controlled, double-masked, multicenter phase II study. Diabetes Care. 2010;33(11):2399-2405. doi:10.2337/dc10-0493

26. Gonzalez VH, Campbell J, Holekamp NM, et al. Early and long-term responses to anti-vascular endothelial growth factor therapy in diabetic macular edema: analysis of protocol i data. Am J Ophthalmol. 2016;172:72-79. doi:10.1016/j.ajo.2016.09.012
Clinical Ophthalmology

\section{Publish your work in this journal}

Clinical Ophthalmology is an international, peer-reviewed journal covering all subspecialties within ophthalmology. Key topics include: Optometry; Visual science; Pharmacology and drug therapy in eye diseases; Basic Sciences; Primary and Secondary eye care; Patient Safety and Quality of Care Improvements. This journal is indexed on PubMed
Central and CAS, and is the official journal of The Society of Clinical Ophthalmology (SCO). The manuscript management system is completely online and includes a very quick and fair peer-review system, which is all easy to use. Visit http://www.dovepress.com/ testimonials.php to read real quotes from published authors. 